\title{
Structural Characterization of the Interaction of the Fibroblast Growth Factor Receptor with a Small Molecule Allosteric Inhibitor
}

\author{
Franziska Kappert, ${ }^{[a]}$ Sridhar Sreeramulu, ${ }_{1}^{[a]}$ Hendrik R. A. Jonker, ${ }^{[a]}$ Christian Richter $_{,}^{[a]}$ \\ Vladimir V. Rogov, ${ }_{1}^{[\mathrm{cc}]}$ Ewgenij Proschak, ${ }^{[\mathrm{d}]}$ Bruno Hargittay, ${ }^{[\mathrm{a}]}$ Krishna Saxena, ${ }^{[\mathrm{a}, \mathrm{b}]}$ and \\ Harald Schwalbe ${ }^{*[a, b]}$
}

\begin{abstract}
The interaction of fibroblast growth factors (FGFs) with their fibroblast growth factor receptors (FGFRs) are important in the signaling network of cell growth and development. SSR128129E (SSR), ${ }^{[1,2]}$ a ligand of small molecular weight with potential anti-cancer properties, acts allosterically on the extracellular domains of FGFRs. Up to now, the structural basis of SSR binding to the D3 domain of FGFR remained elusive. This work reports the structural characterization of the interaction of SSR with one specific receptor, FGFR3, by NMR spectroscopy. This information provides a basis for rational drug design for allosteric FGFR inhibitors.
\end{abstract}

Receptor tyrosine kinases (RTKs) represent the primary mediators that transmit extracellular signals into the cell. Deregulation of their signaling network can result in cancer and other diseases. ${ }^{[3,4]}$ Hence, RTKs and their subfamily, the fibroblast growth factor receptors (FGFRs) form a major class of drug targets. Whereas antibodies target RTKs at the extracellular hormone binding site (orthosteric inhibition, Figure 1A), small molecules intracellularly inhibit the tyrosine kinase activity. Blocking of all signaling pathways is characteristic for both inhibition pathways of the receptor..$^{[5]}$ We recently reported ${ }^{[1,2]} \mathrm{a}$

[a] Dr. F. Kappert, Dr. S. Sreeramulu, Dr. H. R. A. Jonker, Dr. C. Richter,

B. Hargittay, Dr. K. Saxena, Prof. Dr. H. Schwalbe

Goethe University

Center for Biomolecular Magnetic Resonance (BMRZ)

Institute for Organic Chemistry and Chemical Biology

Max von Laue-Straße 7, 60438 Frankfurt am Main (Germany)

E-mail:schwalbe@nmr.uni-frankfurt.de

[b] Dr. K. Saxena, Prof. Dr. H. Schwalbe

German Cancer Research Center (DKFZ)

Partner facility Frankfurt/Mainz

[c] Dr. V. V. Rogov

Goethe University

Center for Biomolecular Magnetic Resonance (BMRZ)

Institute for Biophysical Chemistry

Max von Laue-Straße 9, 60438 Frankfurt am Main (Germany)

[d] Prof. Dr. E. Proschak

Goethe University

Institute of Pharmaceutical Chemistry

Max von Laue-Straße 9, 60438 Frankfurt (Germany)

$\square$ Supporting information and the ORCID identification number(s) for the au-

(13) thor(s) of this article can be found under:

https://doi.org/10.1002/chem.201801770.
multi-FGFR antagonist, SSR128129E ${ }^{[1]}$ (short: SSR, Figure 1 A), an in vivo active compound that acts allosterically on the extracellular D3 domain of FGFR. In a pathway specific manner, SSR inhibits ERK $1 / 2$ activation, whereas PLC- $\gamma$ phosphorylation is not affected. ${ }^{[2]}$ The development of pathway-selective allosteric drugs has several advantages over traditional orthosteric drugs, in particular with regard to selectivity and safety. ${ }^{[6]}$ Up to now, detailed structural studies of the SSR-FGFR interaction have been challenging: No electron density could be observed for a close SSR derivative in the FGFR2D2D3-FGF1-ligand complex X-ray structure. ${ }^{[2]}$ The NMR spectra of the D3 domains of three different isoforms of the FGF receptor (FGFR1, 2, and 4) within a larger construct, but also in isolation, were characteristic for molten globule (MG) states, evidenced by substantial line broadening (see the Supporting Information, S1). ${ }^{[2]}$

Molten-globule behavior demonstrates the existence of secondary structure elements with slowly fluctuating tertiary organization, which makes characterization by NMR spectroscopy close to impossible. ${ }^{[8-11]}$

Previous work revealed SSR binding to the D3 domain. A structural atomistic model could, however, only be derived from MD simulations of FGFR2. ${ }^{[2]}$ The simulations predicted a binding mechanism according to which SSR initially binds to the surface of FGFR2-D3, leading to the stabilization of the short and flexible $\alpha 1$-helix and opening a cryptic binding site.

A recent study ${ }^{[12]}$ of the FGFR3CD3 domain showed a well-resolved ${ }^{1} \mathrm{H},{ }^{15} \mathrm{~N}-\mathrm{HSQC}$ spectrum indicating a stable structure and its amenability to solution NMR studies. Although not all amino acids of the D3 domain are conserved in the different FGFR homologues (see the Supporting Information, S2), we structurally characterized the interaction of SSR to FGFR3CD3. Utilizing the published buffer conditions, FGFR3CD3 remained stable only for a few hours. Buffer screening allowed us to increase the stability of FGFR3CD3 to seven days (see the Supporting Information, S3) at a $\mathrm{pH}$ value of 5.8, but interactions of the protein and ligand were also observed at $\mathrm{pH} 7.5$. Based on 3D experiments and 14 different ${ }^{15} \mathrm{~N}$-amino acid-selectively labeled samples, $71 \%$ of the non-proline protein backbone NMR signals were assigned (see the Supporting Information, S4). Residual conformational dynamics in the intermediate exchange time regime led to disappearing signals localized between P283 and K310. For two segments (Q256-V266 and A314-T317 residing in the $\beta C^{\prime}-\beta E$ loop), additional signals are detected in the apo state of FGFR3CD3, which indicate the presence of two conformational states. Both conformations 

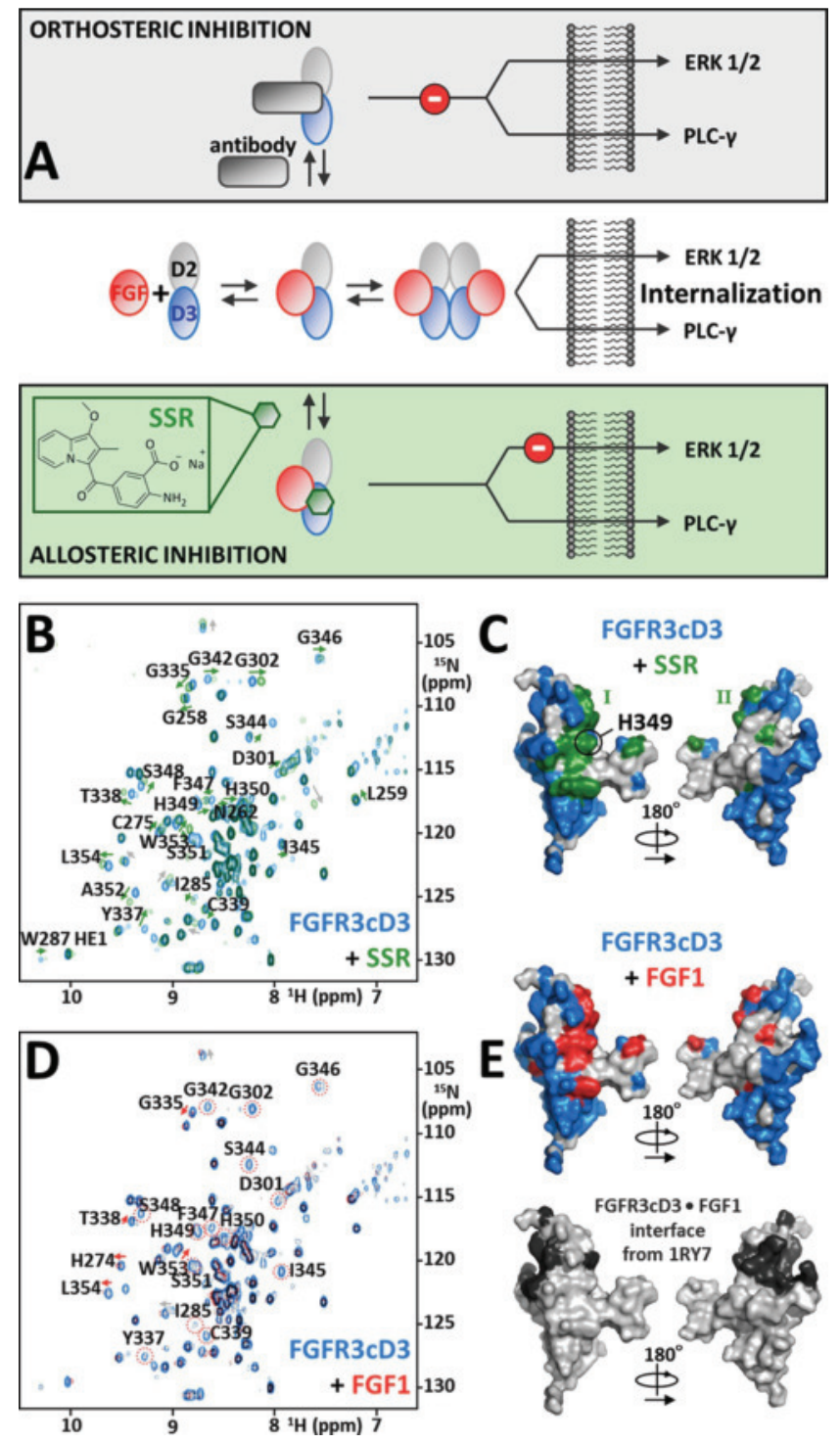

Figure 1. (A) Schematic representation of the FGFR signaling and inhibition mechanisms. Binding of FGF to its receptor leads to receptor dimerization and signal transduction. Inhibition of FGFR signaling utilizing antibodies blocks all signaling pathways. SSR128129E as multi-FGFR inhibitor acts allosterically on the extracellular domains. SSR inhibits ERK1/2 activation selectively. (B) Overlay of the ${ }^{1} \mathrm{H}^{15} \mathrm{~N}-\mathrm{HSQC}$ spectra of FGFR3CD3-apo (blue) and in complex with SSR (green) shows chemical shift perturbations (green arrows for assigned residues; grey arrows for unassigned residues). (C) Mapping of CSPs (in green) on to the FGFR3c crystal structure $\left(1 r y 7^{[7]}\right.$ ) clustered into two regions: the main SSR interaction site (region I) and the area overlapping with the FGF hormone binding site (region II). All the backbone assigned residues are colored in blue and the unassigned ones in grey. (D) Overlay of the ${ }^{1} \mathrm{H}^{15} \mathrm{~N}$-HSQC spectra of FGFR3CD3-apo (blue) and in complex with unlabeled FGF1 (red). Upon interaction with FGF1, the signals disappearing (dotted circles) and showing CSPs (arrows) are indicated. (E) (top) Mapping of FGF1-induced effects onto the surface of FGFR3c (in red). (below) The residues in the direct interface of FGFR3CD3-FGF1 derived from the crystal structure are highlighted in black.

could be assigned and one of the two conformations is indicative for random coil behavior. Addition of SSR had no impact on this conformational heterogeneity (see the Supporting Information, S5), but induced 28 observable chemical shift perturbations (CSPs, Figure 1B) to FGFR3CD3 with a $K_{\mathrm{D}}$ value of
$49 \mu \mathrm{M}$ (2D NMR), in agreement with the $K_{\mathrm{D}}$ of SSR binding to FGFR1D1D2D3-FGF1. ${ }^{[2]}$ The binding site of SSR is visualized on the surface of the FGFR3CD3 crystal structure (PDB: 1ry7; ${ }^{[7]}$ Figure $1 \mathrm{C}$ and the Supporting Information, S6). The induced CSPs are confirmed by NMR of amino acid-selectively labeled samples (Supporting Information, S7). The CSPs spread across two regions. The main and largest region exhibits the strongest CSPs (region I, Figure 1C, left), whereas the second region is positioned on the opposite side of D3 and overlaps with the FGF binding surface (region II, Figure $1 \mathrm{C}$, right). Because the assignment of FGFR3CD3 is not complete, a large region (P283-K310) could only be partially analyzed for SSR interaction (Supporting Information, S4 and S6). The dynamic nature of this region is in line with high B-factors (residues 288-308; Supporting Information, S8) in the crystal structure (1 ry $7^{[7]}$ ).

To further distinguish between both FGFR3CD3 regions affected by SSR and its suggested allosteric mechanism, we investigated the FGFR3CD3-FGF1 complex. We focused our studies on the D3 domain, which binds SSR in a $\mu$ M-regime compared to a mM binding of the D2 domain. ${ }^{[2]}$ An overlay of the ${ }^{1} \mathrm{H},{ }^{15} \mathrm{~N}-\mathrm{HSQC}$ spectra of FGFR3CD3 with and without unlabeled FGF1 (Figure 1D) reveals effects on residues that are part of the FGFR3CD3-FGF1 direct interface and other residues (e.g., G302, F347, S348, H349, W353, L354), that are also involved in the SSR binding to the D3 domain. A subset of peaks in the D3 domain that disappear upon binding of FGF1, reappear upon addition of SSR (e.g., S344, G346, S348, L354) indicating that SSR might modulate the complex by weakening the FGFR3CD3-FGF1 interaction (Supporting Information, S9). However, SSR addition shows only a slight influence on FGF1 within the complex (Supporting Information, S10). We thus conclude that SSR is bound by FGFR3CD3 both in the presence and absence of FGF.

To further substantiate that the allosteric region represents the binding epitope for SSR on FGFR3CD3, we performed sitedirected mutagenesis studies (criteria for selection of residues (Y337, S348, H349, and S351), which are summarized in the Supporting Information, S11). Additionally, the mutation of two residues served as control (I285-FGF binding region; H327lowest CSP; see S11). The structural integrity of all mutants was monitored by $2 \mathrm{D}{ }^{1} \mathrm{H},{ }^{15} \mathrm{~N}$ experiments $(\mathrm{S} 12)$ and $K_{\mathrm{D}}$ values were determined (S13 and S14). NMR titration revealed that only the mutation of $\mathrm{H} 349$, part of the here proposed allosteric binding site, significantly changes the $K_{\mathrm{D}}$ value of SSR to the target protein. Mutation of the histidine's aromatic and polar character to a non-aromatic system as, alanine or aspartate or lysine resulted in an over twenty-fold higher $K_{\mathrm{D}}$ value $(\mathrm{H} 349 \mathrm{~A}$, $\mathrm{H} 349 \mathrm{D}, \mathrm{H} 349 \mathrm{~K}>1000 \mu \mathrm{M}$; Figure $2 \mathrm{~A}, \mathrm{~S} 13)$ than the wild-type $(49 \mu \mathrm{M})$, whereas all other mutants except S351 (171 $\mu \mathrm{M})$ behave as the wild-type (52-79 $\mu \mathrm{M}, \mathrm{S} 14)$. We deduced H349 to be the hot spot for SSR interaction confirming this region as allosteric SSR binding site on D3. H349 was further mutated to phenylalanine and tyrosine, which are conserved in FGFR2 and 4, respectively. Both mutants $\mathrm{H} 349 \mathrm{~F}$ and $\mathrm{H} 349 \mathrm{Y}$ retained the $\pi-\pi$ interaction, resulting in higher affinities (H349F:214 $\mu \mathrm{m}$; H349Y:118 $\mu \mathrm{M}$ ) for SSR binding than the other mutants (H349A, H349D, and H349K). Therefore, we assume that, apart 
A

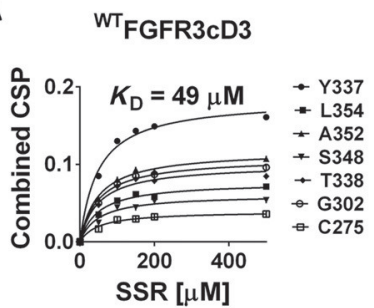

B

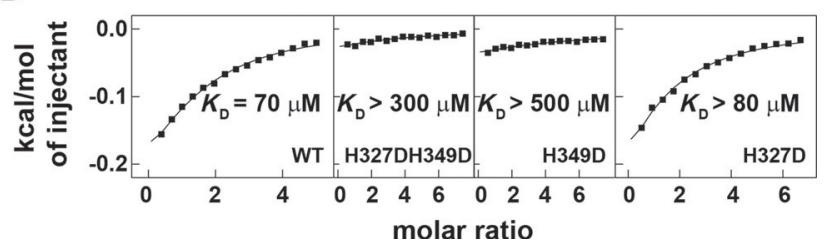

Figure 2. (A) NMR-based (CSP) titration curve for the interaction of SSR with

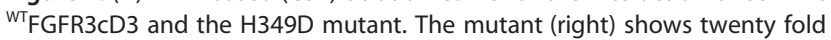
reduced affinity to SSR in comparison to the wild-type receptor (left). (B) ITCbased titration curves and $K_{\mathrm{D}}$ values for the interaction of SSR with

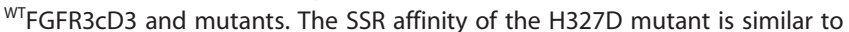
wild-type, while the H349D mutant and H327DH349D double mutant exhibit at least a five-fold higher $K_{\mathrm{D}}$ value.

from the aromatic system, the polar properties of the histidine side chain are required for the SSR-FGFR3CD3 interaction. Furthermore, using differential scanning fluorimetry (DSF) the protein melting temperature $\left(T_{m}\right)$ of FGFR3cD3-apo, FGFR3cD3-SSR complex, and the mutants thereof were determined. Whereas SSR binding to wild-type protein induces a similar increase in melting temperature $\left(\Delta T_{\mathrm{m}}\right)$ as the single point mutation of $\mathrm{H} 349\left(T_{\text {mwt }}-T_{\text {mapo }}=+5.4^{\circ} \mathrm{C} ; \mathrm{S} 15 \mathrm{~B}\right)$, all other mutants reveal either no effect or a decrease in melting temperature (S14). The increased protein stability of the H349A mutant, together with its higher $K_{\mathrm{D}}$ value for SSR binding, supports $\mathrm{H} 349$ to be a key amino acid in the FGFR3CD3 interaction with SSR (S14).

Isothermal titration calorimetry (ITC) confirmed the NMR determined affinities of FGFR3CD3 wild-type and its mutants to the SSR inhibitor despite a high noise level (S16 and S17). The FGFR3CD3 wild-type and its H327D mutant revealed a $\mu \mathrm{M}-$ binding (Figure 2B). In contrast, the H349D FGFR3cD3 mutant and its H327DH349D double mutant do not show binding to SSR. Due to the low solubility of SSR in aqueous buffers, we used a titration scheme with lower injection numbers but higher volume of single injections to observe the small molar enthalpy of the interaction. The FGFR3CD3-SSR titration profile shows a very low binding enthalpy, almost at the edge of detection and yields a $K_{\mathrm{D}}$ in the 50-100 $\mu \mathrm{M}$ range, confirming the $K_{\mathrm{D}}$ value determined by NMR. The low binding enthalpy $(\Delta H=$ $-0.5 \mathrm{kcal} \mathrm{mol}^{-1}$ ) indicates that the FGFR3cD3-SSR interaction occurs by means of association of hydrophobic surfaces on FGFR3CD3 and SSR molecules, with a minor contribution of enthalpy-driven interactions. Significant positive entropy changes upon SSR binding turns out to be the dominant component to the Gibbs free energy $(\Delta G)$ of binding. As the NMR spectra of the amide signals do not indicate increased dynamics (backbone hydrogen bonding and $\beta$-sheet secondary structure do not change), it most probably involves the amino acid side- chains and the hydrophobic effect. In agreement, the FGFR3CD3 mutations (H327DH349D and H349D) which disrupt the hydrophobic binding surface, also weaken the FGFR3CD3SSR interaction, yielding an ITC profile not distinguishable from the SSR dilution heat.

We examined the influence of the SSR-fragments on FGFR3CD3. Addition of neither 5-acetyl-2-aminobenzoic acid nor its simpler derivative 2-aminobenzoic acid induced CSPs (S18). In contrast, the addition of the 1-methoxy-2-methylindolizine-fragment to FGFR3CD3 induced several CSPs (G302, Y337, T338, I345, H349, S351, A352, W353, L354 (S18C)) indicating that this fragment interacts with the proposed allosteric binding site. Furthermore, H349 displays the strongest CSP (S18C).

To generate an inhibitor binding model based on the NMR CSPs and mutagenesis data, we performed HADDOCK calculations ${ }^{[14]}$ utilizing the FGFR3c crystal structure (1ry7), although it contains several challenges (low resolution, no waters, locally increased B factors, artificial stabilizing crystal contacts). The alignment of 13 published FGFRD2D3-FGF crystal structures depicts a flexible loop around residues 288-308 of all D3 domains (S19), supporting our NMR observations. Therefore, this region was left flexible during all stages of the docking. The ambiguous interaction restraints (AIRs) were defined for the surface exposed residues that exhibit the largest CSPs (D301, G302, F347, H349, H350, S351, W353 and N262) and also include the disappearing signal of the R252 sidechain (Figure $3 \mathrm{~A}$ ). From the fragment analysis, an additional ambiguous restraint was included between the H349 sidechain and the SSR indolizine moiety. The docking resulted in a large main cluster (Figure $3 \mathrm{~A}$ ) containing the lowest energy structures that represents an allosteric binding site on the D3 domain (S19) consistent with the CSPs, mutagenesis and X-ray structural data (Figure 1C, S6) and a smaller minor cluster (S20). Both poses indicate the same potential binding site for SSR and show a stacking of the SSR indolizine with the H349 sidechain. A subsequent energy minimization performed using Molecular Operating Environment ${ }^{[15]}$ (MOE) suggested a binding pose in congruence with the major HADDOCK binding pose. SSR interacts strongly with the amino acids T303, T338, H349, H350, S351, D301, and G302 (Figure 3B) in the SSR-binding site of FGFR3CD3, further highlighting the importance of this binding surface.

This approach yielded a binding mode consistent with NMR data modeled onto available crystal structures (S19). We previously $^{[2]}$ reported a significant effect of SSR on the conformational landscape of the receptor, proposing a binding model to the D3 domain of FGFR, involving a newly exposed cavity within this domain. In contrast, the binding site determined in this study is localized on to the surface of the D3 domain and is supported by NMR, ITC, and biological data of H349 mutants.

Interestingly, the SSR binding site proposed by NMR overlaps partially (residues: D301, T338, F347, and H349) with the binding site of the FGF8b hormone ${ }^{[13]}$ and the klotho proteins on FGFR (Figure 3C). Structurally, unlike all other FGFs, the FGF8 subfamily is composed of a globular domain, common 

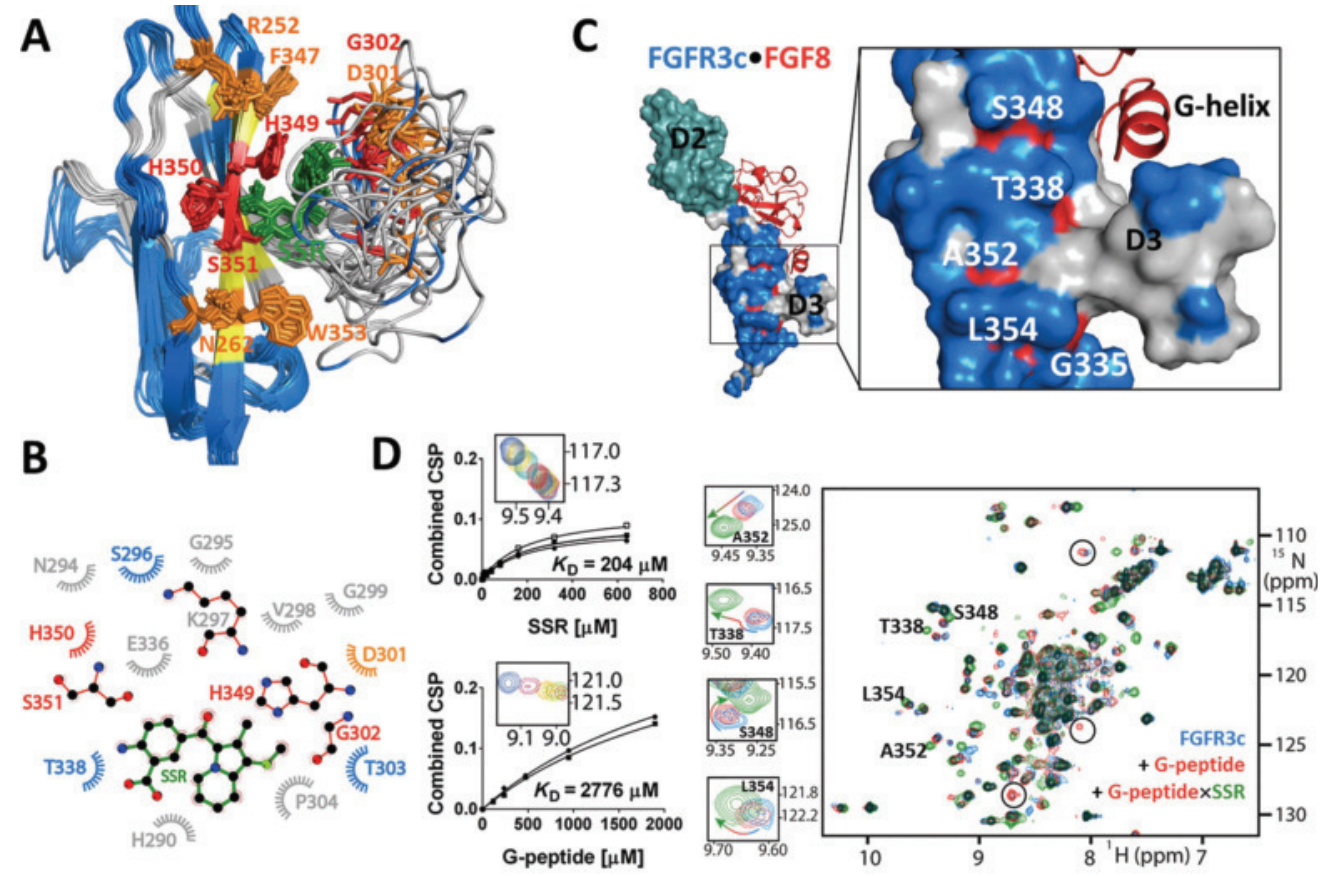

Figure 3. (A) High ambiguity driven docking (HADDOCK) derived potential binding model for SSR (green) on FGFR3CD3 (strongest CSPs in red: G302, H349, H350, and S351, medium CSPs in orange: D301, F347, W353, N262 and as well for residue R252 whose sidechain signal disappears, not surface exposed CSPs in yellow: Y337, T338, G342, S348, A352 and L354, not-assigned residues are indicated in grey). The FGFR3cD3 loop region (residues 288-308) and the SSR ligand were defined fully flexible at all refinement stages and the sidechains at the interface (including those which exhibited large CSPs but were not surface exposed) were allowed to move during the semi-flexible refinement stage. (B) Ligplot showing residues involved in the interaction with SSR derived after MOE-based energy minimization of the HADDOCK model (color coding is similar to that of A). (C) Structural model of the FGF8b-FGFR3c complex. The structure was modeled by superimposing the ligand-binding D2-D3 domains of FGFR3c from the FGF1-FGFR3C crystal structure (1RY7) onto the D2-D3 domains of FGFR2c in the FGF8b-FGFR2c crystal structure (2FDB). Residues for which the backbone amides are assigned are colored in blue and those unassigned in grey. The residues (S348, T338, A352, L354, and G335) of the FGFR3CD3 showing CSPs upon binding of SSR or the FGF8b derived G-peptide are colored in red. (D) Interaction of SSR and the FGF8b derived G-peptide with FGFR3CD3 monitored by NMR. (left, top) SSR binds to FGFR3cD3 with a $K_{\mathrm{D}}$ of $204 \mu \mathrm{M}$ at $\mathrm{pH}$ 7.5. The inset shows a shifting amide crosspeak with varying concentrations of SSR. (left, bottom) G-peptide binds to FGFR3cD3 with a $K_{\mathrm{D}}$ of $2776 \mu \mathrm{M}$ at $\mathrm{pH}$ 8.0. The inset shows a shifting amide crosspeak with varying concentrations of the G-peptide. (right) SSR competes out the G-peptide: ${ }^{1} \mathrm{H}$, ${ }^{15} \mathrm{~N}$-HSQC of FGFR3CD3 apo (blue), in the presence of G-peptide (red) and in the presence of both G-peptide and SSR (green). Addition of G-peptide to FGFR3cD3 results in CSPs (labeled A352, T338, S348, and L354 and shown as zoomed inset) and peaks which appear or increase in intensity (circled). Furthermore, addition of SSR induces CSPs (a subset of these residues overlap with those involved in G-peptide binding) and out-competes the G-peptide.

for all FGFs, and in addition is characterized by a unique varying length of $\mathrm{N}$-terminal sequence, which regulates the biological activity. The hydrophobic residues of the N-terminal Groove-helix (G-helix), which are conserved within the FGF8 subfamily, engage a hydrophobic groove in the D3 domain of the FGFRc isoforms. This binding site can be detected at high $\mathrm{pH}$ ( $\mathrm{pH}$ 8.0); the binding epitope peptide (G-peptide) from FGF8 binds to FGFR3CD3 at a $K_{\mathrm{D}}$ of $2.8 \mathrm{~mm}$ and induces CSPs (Figure 3D; labeled A352, T338, S348, and L354 and shown as zoomed inset). This weak interaction observed between the FGFR3CD3 and G-peptide can be competed out by the addition of SSR, which binds at a $K_{\mathrm{D}}$ of $204 \mu \mathrm{M}$ at pH 7.5 (Figure $3 D$ ). Furthermore, binding of SSR is independent of the H349 protonation state (double/single) as interactions are observed both at pH5.8 and 7.5. A subset of residues which show CSPs, overlap with those involved in G-peptide binding, providing substantial evidence for the NMR proposed binding site.

SSR selectively inhibits the ERK1/2 phosphorylation of FGFRs, and monitoring of the ERK activation therefore repre- sents an indirect approach to evaluate the effect of SSR on FGFR3cD3 wild-type and mutants. Cellular assays (FGFR3c-FGF signaling) tested a selection of wildtype and mutants (T338A, $\mathrm{H} 349 \mathrm{~A}$ and L354A). While the T338A and L354A mutants cannot be activated by FGF1, H349A and wild-type FGFR3C are stimulated. Upon addition of SSR both constructs show inhibition of this stimulation, which is more significant for the wildtype than the H349A mutant (S21).

In conclusion, the amenability ${ }^{[12]}$ of FGFR3CD3 to NMR spectroscopy allowed us to propose a structural model for the interaction of SSR to FGFR3. This NMR-derived model differs from a previously delineated binding site from MD simulation of the FGFR2 receptor. The competition of SSR with the FGF8b derived G-peptide points towards an orthosteric interaction at this site, which is allosteric to the interaction interface of the common globular domains of all FGFs. The FGF8b binding site has previously been delineated for the FGF receptor $1 \mathrm{c}$ and $2 \mathrm{c}$. Thus, the current work substantiates our understanding of the binding site and of the interference with specific signaling pathways. 


\section{Acknowledgements}

We thank Dr. Federico Comitani, Prof. Dr. Francesco Luigi Gervasio, Dr. Chantal Alcouffe and Dr. Corentin Herbert for insightful discussions during the preparation of this manuscript. The work in the group of H.S. is supported by the German center for translational cancer research (DKTK) and EU-access program iNEXT. H.R.A.J. is supported by grant from the German economic ministry (AiF). The work of V.V.R. is supported by SFB 1177 "Molecular and Functional Characterization of Selective Autophagy", Germany. E.P. is supported by the German Research Foundation (DFG, Heisenberg-Professur PR 1405/4-1). Work at BMRZ is supported by the state of Hesse.

\section{Conflict of interest}

The authors declare no conflict of interest.

Keywords: allostery · FGFR • inhibitor • NMR Spectroscopy SSR128129E

[1] F. Bono, F. De Smet, C. Herbert, K. De Bock, M. Georgiadou, P. Fons, M. Tjwa, C. Alcouffe, A. Ny, M. Bianciotto, B. Jonckx, M. Murakami, A. A. Lanahan, C. Michielsen, D. Sibrac, F. Dol-Gleizes, M. Mazzone, S. Zacchigna, J. P. Herault, C. Fischer, P. Rigon, C. Ruiz de Almodovar, F. Claes, I. Blanc, K. Poesen, J. Zhang, I. Segura, G. Gueguen, M. F. Bordes, D. Lambrechts, R. Broussy, M. van de Wouwer, C. Michaux, T. Shimada, I. Jean, S. Blacher A. Noel, P. Motte, E. Rom, J. M. Rakic, S. Katsuma, P. Schaeffer, A. Yayon, A. Van Schepdael, H. Schwalbe, F. L. Gervasio, G. Carmeliet, J. Rozensky,
M. Dewerchin, M. Simons, A. Christopoulos, J. M. Herbert, P. Carmeliet, Cancer Cell 2013, 23, 477-488.

[2] C. Herbert, U. Schieborr, K. Saxena, J. Juraszek, F. De Smet, C. Alcouffe, M. Bianciotto, G. Saladino, D. Sibrac, D. Kudlinzki, S. Sreeramulu, A. Brown, P. Rigon, J. P. Herault, G. Lassalle, T. L. Blundell, F. Rousseau, A. Gils, J. Schymkowitz, P. Tompa, J. M. Herbert, P. Carmeliet, F. L. Gervasio, H. Schwalbe, F. Bono, Cancer Cell 2013, 23, 489-501.

[3] E. Zwick, J. Bange, A. Ullrich, Trends Mol. Med. 2002, 8, 17-23.

[4] N. Hallinan, S. Finn, S. Cuffe, S. Rafee, K. O. Byrne, K. Gately, CANCER Treat. Rev. 2016, 46, 51-62.

[5] T. Regad, Cancers 2015, 7, 1758-1784.

[6] A. Christopoulos, Nat. Rev. Drug Discovery 2002, 1, 198-210.

[7] S. K. Olsen, O. A. Ibrahimi, A. Raucci, F. Zhang, A. V. Eliseenkova, A. Yayon, C. Basilico, R. J. Linhardt, J. Schlessinger, M. Mohammadi, Proc. Natl. Acad. Sci. USA 2004, 101, 935-940.

[8] C. Redfield, Methods 2004, 34, 121-132.

[9] K. Kuwajima, Proc. Indian Nat. Sci. Acad. 2002, 68, 330-340.

[10] S. Ramboarina, C. Redfield, J. Mol. Biol. 2003, 330, 1177-1188.

[11] D. A. Dolgikh, R. I. Gilmanshin, E. V. Brazhnikov, V. E. Bychkova, G. V. Semisotnov, FEBS Lett. 1981, 136, 311-315.

[12] J. Kalinina, K. Dutta, D. Ilghari, A. Beenken, R. Goetz, A. V. Eliseenkova, D. Cowburn, M. Mohammadi, Structure 2012, 20, 77-88.

[13] R. Goetz, M. Ohnishi, X. Ding, H. Kurosu, L. Wang, J. Akiyoshi, J. Ma, W. Gai, Y. Sidis, N. Pitteloud, O. M. Kuro, M. S. Razzaque, M. Mohammadi, Mol. Cell. Biol. 2012, 32, 1944-1954.

[14] C. Dominguez, R. Boelens, A. M. J. J. Bonvin, J. Am. Chem. Soc. 2003, $125,1731-1737$.

[15] Molecular Operating Environment (MOE), 2013.08, Chemical Computing Group ULC, Montreal, QC, Canada, 2018.

Manuscript received: April 9, 2018

Accepted manuscript online: April 14, 2018

Version of record online: May 3, 2018 